\title{
Efecto de la pobreza en la degradación de los recursos naturales focales del Parque Nacional Huascarán
}

Effect of poverty in the degradation of natural resources focus Huascaran National Park

Francisco Huerta Berríos ${ }^{1}$ y Denisse Mendoza Riv

\section{RESUMEN}

Esta investigación pretende explicar la relaci $A$ entre pobreza de los hogares que habitan en la Zona de Amortiguamiento (A. VH) y degradación de los recursos naturales focales del Parque Nacional carán ( NH) localizado en la sierra noroccidental del Perú, área natural prote la por el Estado desde 1975 y declarada por la UNESCO Reserva de Biosfera de la Humani ad en 1977 y Patrimonio de la Humanidad en 1985. El estudio se en dar provenientes de la encuesta de visita única a 316 jefes de hogar de la ZA N', ar de mostrar la evidencia del planteamiento teórico, reforzado po $10 \mathrm{~s}$ nteo dentes empíricos de Altieri y Másera. Se halló que la pobreza de los ho qres rererida zona degrada los recursos naturales focales del Parque con la a cuación otidiana de los campesinos pobres de la zona, afectando la estabilidad colos a de lavegetación de los bosques, bosquetes y matorrales que les provee d leña y ma para la construcción; asimismo, a las hierbas medicinales y arom cas andinas, a los peces de agua dulce y los pastos naturales para el ganado. L - halló ros re paldan empíricamente el planteamiento teórico que liga de modo causal la pobre y degradación de los recursos naturales bajo condiciones de subsistencia ponración campesina.

Pat, bras clave: subsistencia; indigencia; degradación; reserva de biósfera; parque nacional.

\section{ABSTRACT}

This research aims to explain the relationship between the buffer Zone household poverty of Huascaran National Park (ZAPNH) and degradation of natural resources focus of

1 Universidad Nacional Santiago Antúnez de Mayolo. Huaraz, Perú. 
Huascaran National Park (PNH) located in the occidental highland of Peru, whose natural area is protected by the state since 1975 and was declared by UNESCO in 1977 Biosphere Reserve Site of the humanity and Natural Heritage of Humanity in 1985. The study was based on data from the single-visit survey to 316 heads of ZAPNH home, to show evidence of the theoretical approach of Chayanov (1974), reinforced by the empirical record of Altieri and Másera (1997), (Short et al., 2002), Mendez (2003), (De La Paz et al., 2005) and Martinez (1991). It was found that the poverty of the households in that area directly affect the degradation of focal natural resources of the park, through the everyday actions of the poor rural housed in the study area that affect ecological stability, as is the case of forest vegetatio, thicket and brush that provides them with firewood and building materials, also med inal an aromatic herbs, fish of sweet water and natural pasture for the lives ck. 1 fineming support empirically the theory of the causal link between pover, and de adation of natural resources under subsistence conditions of the rur apulat

Keywords: subsistence; poverty; degradation; biospher feser ational park.

\section{ICHIKLLACHAW}

Kay musyapakuyqa tsuyantsaatsimun imanaw i wayikunachaw waktsa

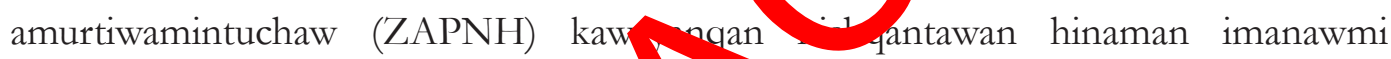
llapan hirkachaw kaq Parki Nasiyun y an (PNH) hutiyuq nishqanchaw ushakaatsishqantam, kayqa Per pa hinc kunti kaqmanmi karan, kay hirkaqa Peru pushaq umalliqmi 1975 pats roig washa, hinaman UNESCOpis 1977 watachaw runakunapa taapaynin ni rqa, h. aman llapan runakunapa hatun kapuynin 1985chaw patsaatsishqan. Kay mu apakuy ashipakuyqa tapukuykuna nishqankunawanmi rurakashqa, tsayp a huk ku llam aywakashqa 316 ZAPNHpa qatiqninkunaman watukakashqa. Tsay an yachay nishqanta Chayanoc (1974chaw) ninqannaw, hinamanpi - vayky a Altieriwan Másera (1997), Méndez (2003), De La Paz (2005) Martít ez (19, niyanqanta yanapayan. Wayikunapa waktsanchaw tarikashqa imanawn arkintsikpa hirkankunachaw patsaypa llapan kaqta ushakaatsiyan, kaykuna ushakaatsay kikin tuna hirkachaw waktsakuna taakuqkunam kayan, kaykunam llapan hachakunata, tuturakunata mana alliyaatsiyan, kay llapan hachakunam yamtanpis, qirunpis rurayaananpaq qun, hinamanpis hirkakunachaw hampi pukutaq qurakunata, challwakunatapis ushakaatsiyan, uushakunapa pastuntapis. Kay musyapakuyqa yuyay, yachay kanqantam yanapakun, imanawpis waktsayaynintawan llapan hirkachaw kaynintam tinkutsin, tsaynawpa imanawllapis tuna runantsik kawar qatiyaananpaq.

Pushaq shimikuna: imanawllapis tura runakunapa kawaynin; kaqpa ushakaatsaynin; kawaypa waataynin; parki nasiyunal nishqan. 


\section{INTRODUCCIÓN}

Los elevados niveles de pobreza rural y la degradación de los recursos naturales son probablemente dos de los principales desafíos que enfrenta la sierra de Áncash. Entender la interacción entre ambas situaciones es substancial si se pretende diseñar políticas que combinen de manera armoniosa los objetivos económicos, sociales y ambientales. La presente investigación busca contribuir a la explicación del nexo entre ambos fenómenos presentes en el referido Parque, a partir del análisis de los datos captados mediante una Encuesta previamente validada, aplicada en visita única a una muestra seleccionada al azar entre los jefes de hogar de la Zona de Amortiguaminato del Parque Nacional Huascarán. Entiéndase por «degradación» la pérdida de c pacidad el suelo o del agua, o de otros recursos, para sustentar una determinada acti dad prod activa en un determinado nivel. Es un concepto referente a la pérdida cep du biofísica de un recurso natural (Morales, 2005: 30).

La base teórica de este trabajo corresponde a Chaya (1974, qu desde la perspectiva de la teoría de la economía campesina, razona que Los pronos productores campesinos implementan un conjunto de estrateg sorien as a asegurar la sobrevivencia del grupo familiar, dado que cuentan con oca erra, do mala calidad, pero abundante fuerza de trabajo. En tales condiciones bjetivo rincipal es sobrevivir y no maximizar ganancias, lo que implica aprovec ar al máximo los escasos recursos disponibles de baja productividad y frágiles. La pres ún a los pue son sometidos sobrepasa la capacidad normal de recuperación ôs suelu la cubierta vegetal».

Altieri y Másera (1997), Kustud b 200z), (Short et al., 2002), (De La Paz et al., 2003), Torres y Vergara (s.f) Mén lez ( 03), Hernández (1994) y Bartra (2010) añaden que la dirección de las ro cio a consalidad entre pobreza y estado de conserva de los recursos na rales y mbientales es compleja y no necesariamente lineal; pero que en lo sustan wo s muchos los casos en que claramente la pobreza causa degradación y la degr dación, po eza. También Martínez (1991) es partícipe de este razonamiento, qu n ha $\delta$ en sus estudios empíricos que «Las prácticas tradicionales incluyen un eymo a basto en zonas comunales, debido a la presión interna y externa de los camp sinos ... y la demanda de combustible destruye 1 os bosques cercanos a 1 os oros y ludades en muchos países. La pérdida de los árboles provoca el incremento erosión. En 1 os lugares en que se utiliza estiércol seco en lugar de leña, se reduce la fertilidad del suelo, y en consecuencia la cosecha (...) La falta de leña es un problema en algunas regiones de América Latina, en las regiones áridas o semiáridas, no sólo en los Andes sino también, por ejemplo, en las zonas costeras de Perú y Chile (...). Las sierras del Perú y de Bolivia son zonas con un alto riesgo de desertificación y degradación y con una aguda escasez de leña» $(63,65-67)$.

El presente trabajo se inicia describiendo la magnitud del problema de la pobreza para el Parque Nacional Huascarán; seguidamente se revisa literatura relevante así como estudios recientes sobre el tema para América Latina y el Perú; luego se introduce el 
modelo conceptual así como la fuente de datos utilizada; posteriormente, se presenta la evidencia econométrica de la relación entre pobreza y degradación de los recursos naturales focales del Parque y finalmente, se extraen las conclusiones e implicaciones de políticas públicas recomendables y posibles líneas de investigación futura.

Para la verificación de las hipótesis se abordó el análisis desde tres líneas: la estimación de la pobreza de la Zona de Amortiguamiento del Parque; la estimación de la degradación de los recursos naturales focales del mismo Parque; y la estimación del modelo logístico que relaciona los indicadores de la pobreza de los hogares y los de la degradación de los recursos naturales focales del espacio geográfico comprendido. tados estadísticos y econométricos confirman las previsiones teóricas y las h partida y coinciden con los antecedentes empíricos en varios países de Amér a Latina

\section{MATERIALES Y MÉTODOS}

El tipo de estudio es el de una investigación empírica, descri tiva, volioutiva, retrospectiva y con metodología mixta, vale decir, cuantitatiy y cua tava. Se aplicó el diseño cuantitativo no experimental, transeccional, co e vional-c usal y probabilístico. Según datos del Censo Nacional de Población y Vivienda el INEI (2009), se estima que la población de la Zona de Amortiguami ato del Parque es de 83,047 habitantes, asentados 61,718 en el área rural (74\%) y 21, 29 en el y bano (26\%). El jefe de hogar constituyó la unidad de análisis. Se estipn que en a población de 19,400 hogares en dicha Zona distribuidos en 612 asent mí de la cual se seleccionó una muestra al azar de 316 hogares, por estrat ural arbano al 95\% de seguridad y con la ayuda de un mapa territorial cuyos res of ciaron de informantes. En la aplicación de la encuesta de visita única se urizo ecnica ue la entrevista. El instrumento de medición se validó con la ayuda axpertos rofesionales del Parque Nacional Huascarán; y su confiabilidad con laprueba Alfa de Crombach, de valor 0,649, para 27 ítems analizados.

La clasificación, nam nto, codificación y el proceso de datos se hizo con ayuda del softy a S SS, on 19.0. El análisis se efectuó, en principio, sobre la base de los indicaa ves variable utilizando el estadístico de Chi cuadrado, el Odds Ratio. Se conside una relación de dependencia significativa si el valor $\mathrm{P}<0.05$. Para el análisis multivariare se estimó un modelo econométrico de regresión logística que advierte los indicadores más significativos de las variables que afectan la degradación de los recursos naturales focales del Parque Nacional. En la regresión múltiple, la variable dependiente es discreta; por esta condición se trata de un modelo útil en frecuentes situaciones prácticas de investigación en que la respuesta toma únicamente dos valores: 1 , presencia (con probabilidad $p$ ) y 0 , ausencia (con probabilidad 1- $p$ ): Los indicadores de la variable explicativa fueron cuantitativos y cualitativos. El Análisis de Regresión tuvo dos propósitos: de un lado, estimar la relación existente entre los indicadores de la variable independiente y los de la respuesta; y, de otro, clasificar a los individuos en el 
grupo expuesto y no expuesto en función de su probabilidad. El modelo de regresión logística múltiple respondió a la siguiente expresión:

$$
\mathrm{Y}=\frac{1}{1+\varepsilon^{-\left(B_{0}+B_{1} X_{1}+\ldots+B_{K} X_{K}\right)}}
$$

Donde:

$\beta_{1,2,3 . . .}$ : Coeficientes de las variables independientes.

Y: Degradación de los recursos naturales focales del Parque Nacional Huascarán.

X: Pobreza de los hogares de la Zona de Amortiguamiento del Par ae N ional Huascarán.

\section{RESULTADOS}

Pobreza del hogar en la Zona de Amortiguamiento renargue cional Huascarán El tamaño del hogar es, en promedio, de 4.2 Aembr el mínimo 1 y máximo 6. El Ingreso económico mensual del hogar, po to conce to, es de S/430.6, en promedio. El ingreso per cápita mensual, S/102 5, equ lente a US\$ 30 por mes/persona. El 65.5\% de los hogares cuenta con ngresos mensuales iguales o menores de S/465, y $34.5 \%$ con más de $\mathrm{S} / 465$.

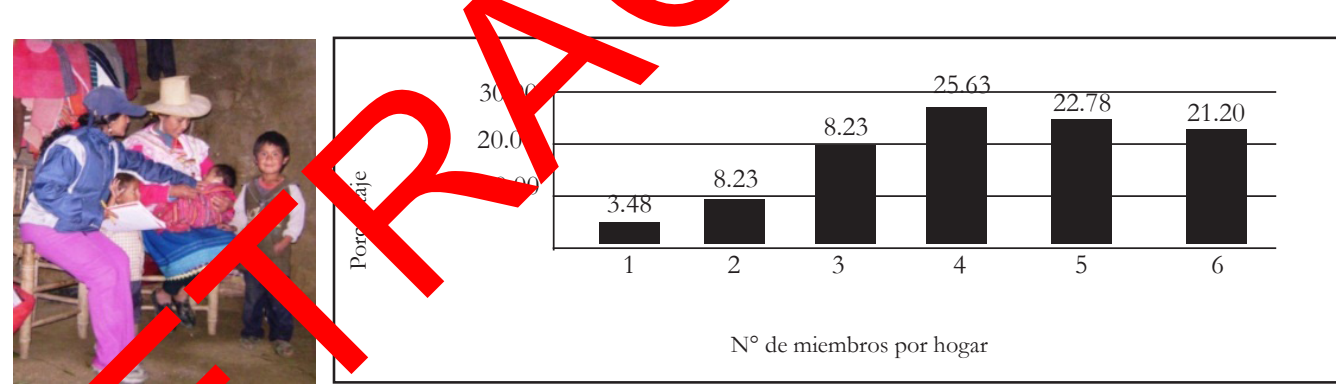

Figura 1. Número de miembros del hogar de la ZAPNH

aram dia de los jefes de hogar encuestados fue de 45 años. El sexo que prevalece ellos es el masculino, con el 85\% del total.

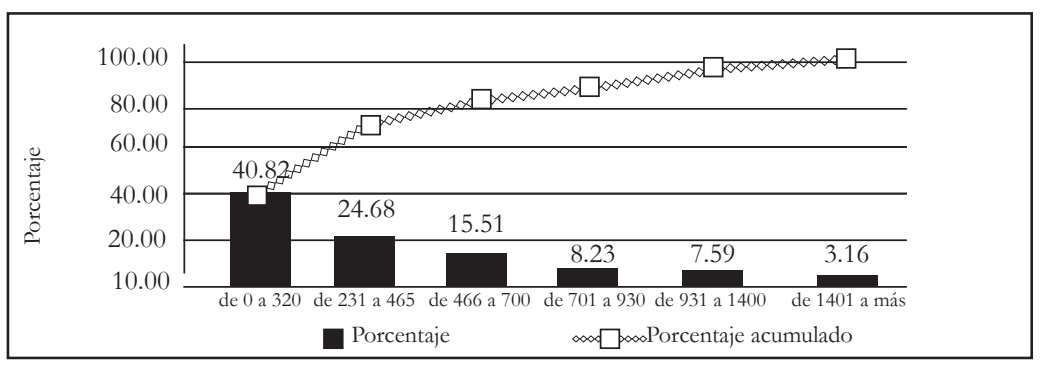

Figura 2. Ingreso económico mensual del hogar de la ZAPNH 
La principal actividad económica de los jefes de hogar es la agropecuaria, con 60,1\%; la segunda más relevante es la prestación de servicios, con 14,2\%.

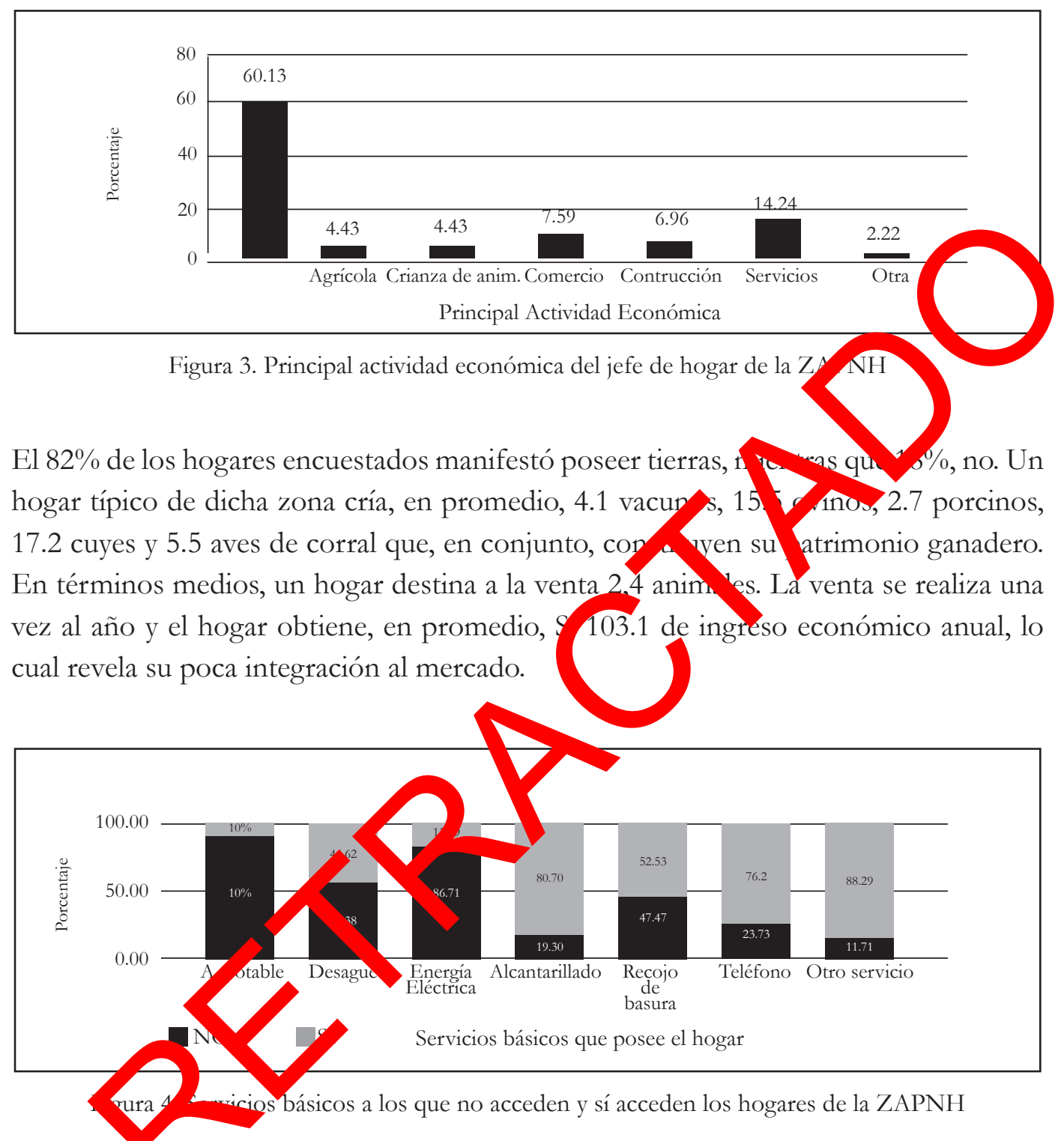

Los servicios básicos de mayor cobertura son el agua potable $(89,2 \%)$; desagüe, $(55,4 \%)$ y energía eléctrica $(86,7 \%)$. Sin embargo, acceden menos a los del alcantarillado $(19,3 \%)$, recojo de la basura (47,5\%), teléfono (23,7\%); y otros servicios $(88.3 \%)$, lo que muestra la situación de pobreza de los hogares de la Zona de Amortiguamiento del Parque. 


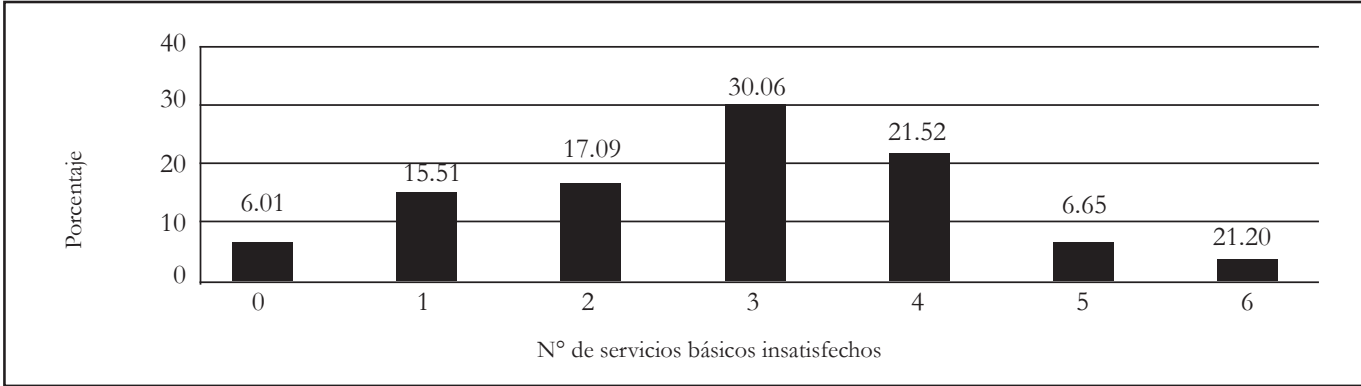

Figura 5. Número de servicios básicos a los que no acceden los hogares de la ZAPNH

El nivel de instrucción de la mayoría de jefes de hogar de la ZAN $\mathrm{VH}$ es 1 primaria $(47,2 \%)$, seguidó de la secundaria $(29,1 \%)$. Solo un $15,5 \%$ ogra n en el superior. Sin embargo, 8,2\% de los jefes de hogar no alcanzaron ingún n rel. La condición laboral mayoritaria de los encuestados, con 15 años más ed. e a de subocupados, con $47.2 \%$, seguida por los desocupados, con 36.7\% V cy empreo adecuado, 16.1\%, hecho que guarda relación con la práctica de agricu a de secano que solo permite labores culturales en una parte del año, e co vez ermite reafirmar la situación precaria de la fuerza laboral, de los bajo ingresos onómicos que impera entre los hogares. El uso agropecuario de la escas tierra es mayormente para los cultivos en limpio $(53.5 \%)$, seguido de pastos naturales $16.8 \%)$, prestales $(7.6 \%)$ y cultivos permanentes $(6.0 \%)$. Solo el $2.9 \%$ de las tie no tion so productivo, situación que evidencia la escasez de tierras aptas para us pe on y el sostenimiento de los animales domésticos; y también de las fo les ta la leña.

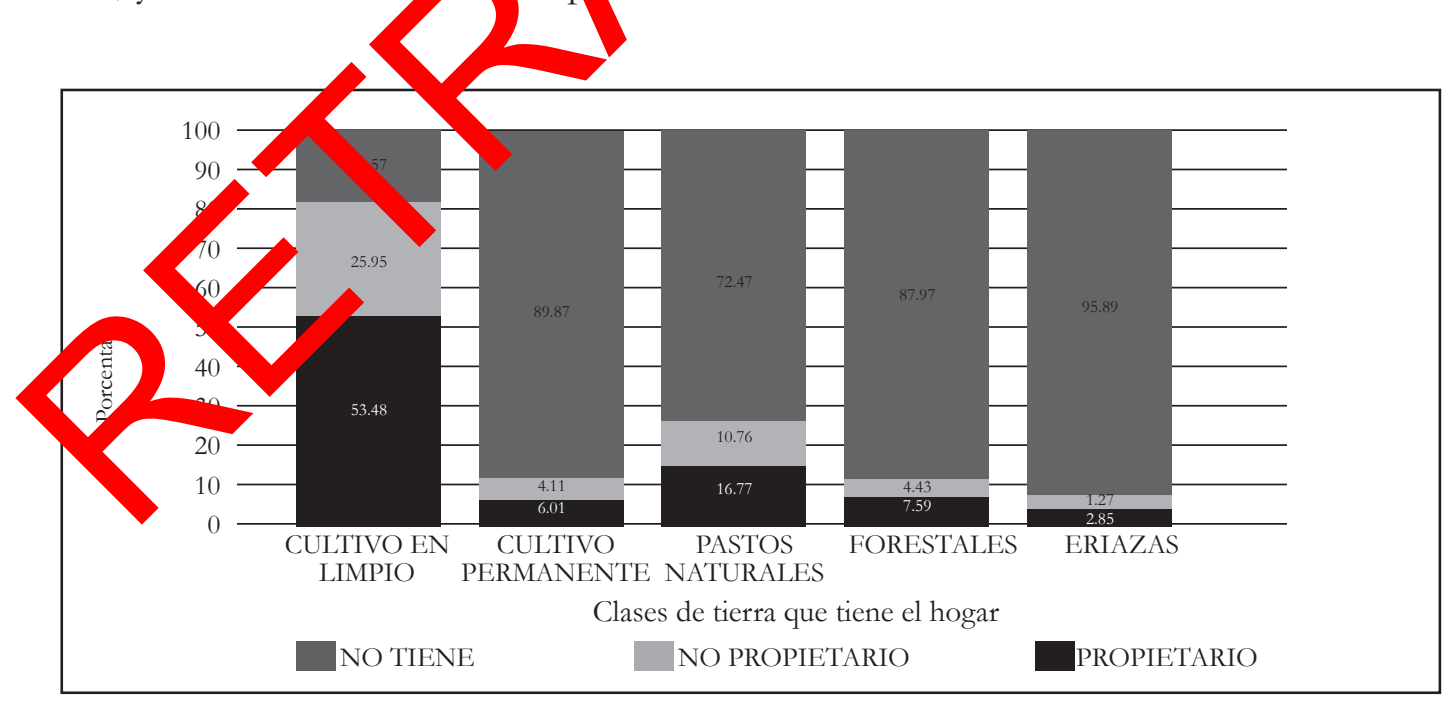

Figura 6. Tenencia de tierra según tipo de uso de los hogares de la ZAPNH 
Estado de los recursos naturales del hogar y del Parque Nacional Huascarán

El 44\% de los jefes de hogar indicó tener la necesidad de pastos del Parque para el pastoreo de sus ganados mayores; de éstos, con una intensidad entre alta y muy alta (28,5\%); aunque $56 \%$ indicó no tener necesidad. La presión por el uso intenso de los pastos naturales es manifiesta y, por consiguiente, el riesgo de su degradación.

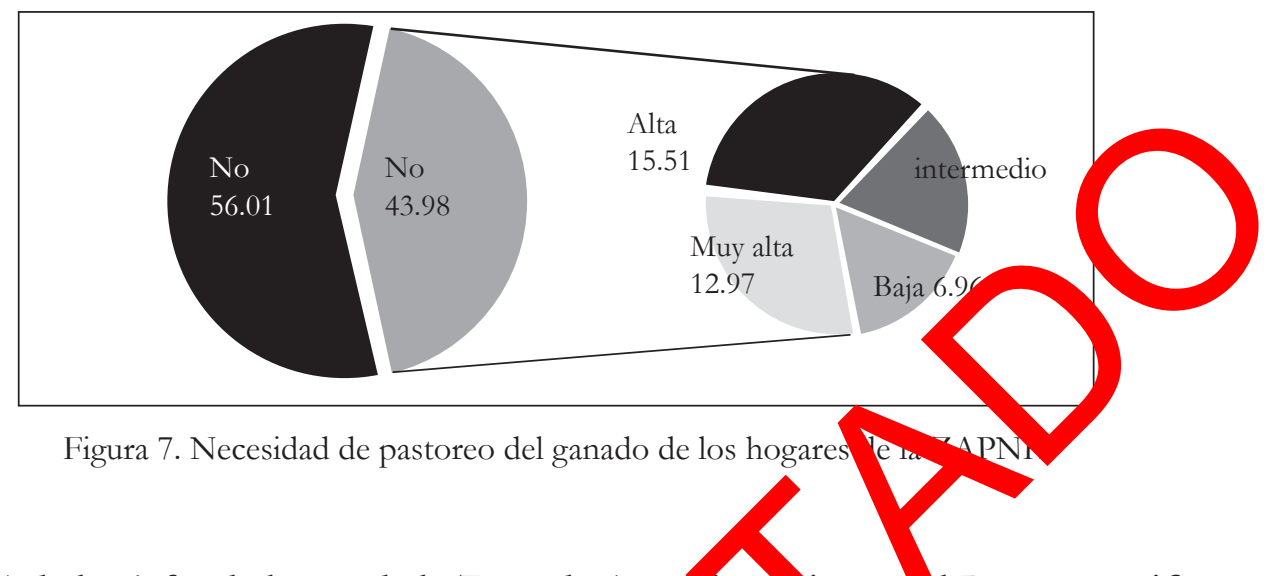

El 55,5\% de los jefes de hogar de la Zona de Amortiguar ento del Parque manifiesta tener necesidad de leña con fines energéticos propios, perono así el 44.5\%. Entre los que usan, 27,6\% tiene una necesidad entr alta y $m$ alta y revela una presión alta sobre los frágiles bosques, árboles, arbustos y vator les, de manera que la elevada demanda de leña por los campesinos pi bamente contribuya a la degradación de la foresta del Parque Nacional Huascarán. no $00 \%$ de los jefes de hogar de la la referida zona dicen tener necesidad de eco) de $\mathrm{C}$ erbas medicinales y aromáticas del Parque Nacional Huascarán, tanto pa la ción de sus alimentos como para medicar a los integrantes del hogar ellos, $6 \%$ recogen las hierbas diariamente y $3,8 \%$ una vez por semana y $26 \%$ un vez mes. En cada recojo se extrae, un promedio, 103 gramos de hierbas medici ales y 71 gr nos de las aromáticas. También en este caso se percibe una presión so e las rágiles hierbas altoandinas del Parque.

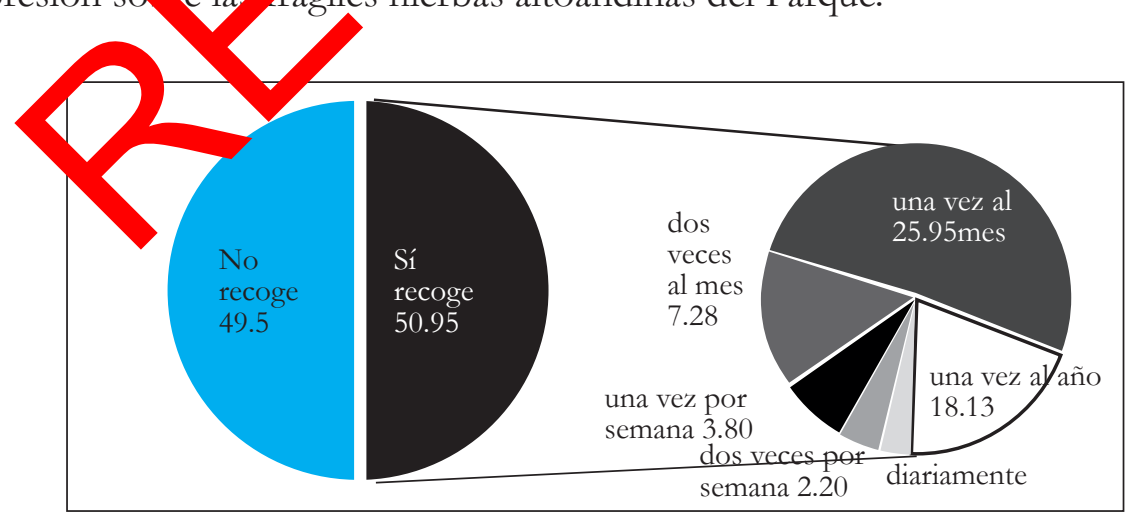

Figura 8. Necesidad y frecuencia de recojo de hierbas medicinales y aromáticas del Parque Nacional por hogares de la Zona de Amortiguamiento 
El 17,1\% de los jefes de hogar indica tener la necesidad de pescar truchas en los ríos o lagunas del Parque Nacional y, de ellos, el 1,6\% dice extraer una vez por semana y $9,2 \%$, una vez al mes, aunque muchos de ellos dijeron que había poca existencia de peces y no como antes.

\section{Modelo logístico estimado}

El modelo de regresión logística múltiple estimado, para determinar la mababilidad de degradación de los recursos naturales focales del $\mathrm{PNH}$, es el siguie te:

Donde:

$$
P=\frac{1}{1+e^{-Z}}
$$

$Z=2,312-1,180 *$ (de 231 a 465)-1,448* (de 466 a 700$)-2,035 *$ (de 701 a 930)-2,649* (de 931 a 1400)-3,046* (de 1401 a más) + , ,753* (no cucrita con recojo de basura)-1,556*(más de 2 miembros ocupados)-1,66* (de 1 a miembros ocupados) $+0,712^{*}$ (de 1 a 2 miembros desocupados) $+243^{*}$ (miembros desocupados) $+0,142 *$ (de 1 a 32 cabezas) $+1,650 *$ (de 3. ç éza a más)-2,162* (primaria)-2,727* (secundaria) $-2,338 *$ (superior).

En la tabla sigu te se puede observar los coeficientes estimados (B), el estadístico Wald (y ald), el valor (Sig.), el Odds ratio $(\operatorname{Exp}(B)$ con sus respectivos intervalos de confian Los re ultados permiten aseverar que hay algunos que actúan como factor a ri go v s 1) y otros como factor de protección $(\mathrm{OR}<1)$, con sus respectivos n varoude confianza. El parámetro del indicador Ingreso Económico del hogar es ne tivo y significa que el aumento de este ocasiona menor degradación de los recursos naturales focales, esto es, $\mathrm{OR} \widehat{\left(\boldsymbol{\beta}_{1}\right)}<1$. 
Tabla 1. Resultados de la regresión del modelo logístico aplicado al efecto de la pobreza en la degradación de los recursos naturales focales del Parque Nacional Huascarán

\begin{tabular}{|c|c|c|c|c|c|c|c|c|c|}
\hline & & B & E.T. & Wald & $\mathrm{gl}$ & Sig. & $\mathrm{OR}=\mathrm{Ex}-$ & I.C. 95,0 & ara EX- \\
\hline & & & & & & & & Inferior & Superior \\
\hline & Constante & 2,312 & 1,130 & 4,186 & 1 & ,041 & 10,099 & & \\
\hline Ingreso Económico & De 0 a 230 & & & 12,208 & 5 & ,032 & & & \\
\hline & De 231 a 465 & $-3,046$ & 1,257 & 5,872 & 1 & ,015 & ,048 & ,004 & ,559 \\
\hline & De 466 a 700 & $-2,649$ & 1,254 & 4,466 & 1 & ,035 & ,071 & ,006 & ,825 \\
\hline & De 701 a 930 & $-2,035$ & 1,298 & 2,456 & 1 & ,117 & 131 & ,010 & \\
\hline & De 931 a 1400 & $-1,448$ & 1,229 & 1,388 & 1 & 239 & 235 & ,021 & 2,614 \\
\hline & De 1401 a más & $-1,180$ & 1,268 & ,866 & 1 & ,352 & ,307 & ,026 & 3,688 \\
\hline Recojo de basura & No & 753 & ,313 & 5,785 & 1 & ,016 & 2,123 & & \\
\hline Miembros con 15 & Ninguno & & & 13,189 & 2 & ,001 & & & \\
\hline $\begin{array}{l}\text { o más años están } \\
\text { ocupados }\end{array}$ & De 1 a 2 Miremoros & $-1,667$ & 1,433 & 1,353 & 1 & ,245 & 189 & & 3,132 \\
\hline & Más de 2 Miembros & $-1,556$ & ,431 & 13,044 & 1 &, 000 & & & ,491 \\
\hline Miembros con 15 & Ninguno & & & 6,077 & 2 & ,048 & & & \\
\hline $\begin{array}{l}\text { o más años están } \\
\text { desocupados }\end{array}$ & De 1 a 2 Miembros & ,712 & ,318 & 5,020 & 1 & & & & 3,797 \\
\hline & Más de 2 Miembros & 1,043 & ,638 & 2,671 & 1 & & & ,812 & 9,919 \\
\hline & No tiene & & & 10,186 & 2 & & & & \\
\hline Pecuario & De 1 a 32 cabezas & 142 & ,430 & 109 & & & & 496 & 2,680 \\
\hline & De 33 cabezas a más & 1,650 & ,603 & 7,489 & & ,006 & 5,207 & 1,597 & 16,974 \\
\hline Nivel de instrucción & Sin nivel & & & 7,581 & & & & & \\
\hline & Primaria & $-2,162$ & & & & & ,115 & ,014 & ,976 \\
\hline & Secundaria & & & & & ,013 & ,065 & ,008 & ,564 \\
\hline & Superior & & & & 1 & 044 & ,097 & ,010 & 943 \\
\hline
\end{tabular}

El parámetro del indir servic de recojo de basura es positivo y significa que a mayor recojo de lo residuo ólidos domésticos, disminuye la degradación de los recursos naturale ocal del Parque, pues $\mathrm{OR} \widehat{\left(\boldsymbol{\beta}_{2}\right)}=2,123>1$. El parámetro del número de miembros del gar co 15 o más años de edad en situación de ocupados, es negativo $\left(\mathrm{OR}_{3}=4\right.$ 1) e in $\quad$ a que al aumentar el número de miembros del hogar ocupados hay una or angadación de los recursos naturales focales. Para la situación de los miembros 1 hogar de 15 o más años desocupados, los parámetros tienen signo positivo $\left(\mathrm{OR}\left(\widehat{\left.\beta_{4}\right)}=>1\right)\right.$, lo cual significa que conforme aumenta el número de miembros desocupados, también aumenta la degradación de los recursos naturales. En cuanto al nexo con el total de animales criados por el hogar, se halló que a mayor número de animales criados, mayor nivel de degradación de los pastos naturales, así lo indica el signo positivo del parámetro de la variable explicativa $\left(\mathrm{OR} \widehat{\left(\boldsymbol{\beta}_{5}\right)}=>1\right)$, esto es, que en los hogares que crían animales de 1 a 32 cabezas aumenta el riesgo de degradación de los recursos naturales una vez más que en aquellos hogares que no crían animales y en aquellos que crían más de 33 cabezas hace que aumente el riesgo de degradación en 5 
veces, respecto a los hogares que no crían animales.
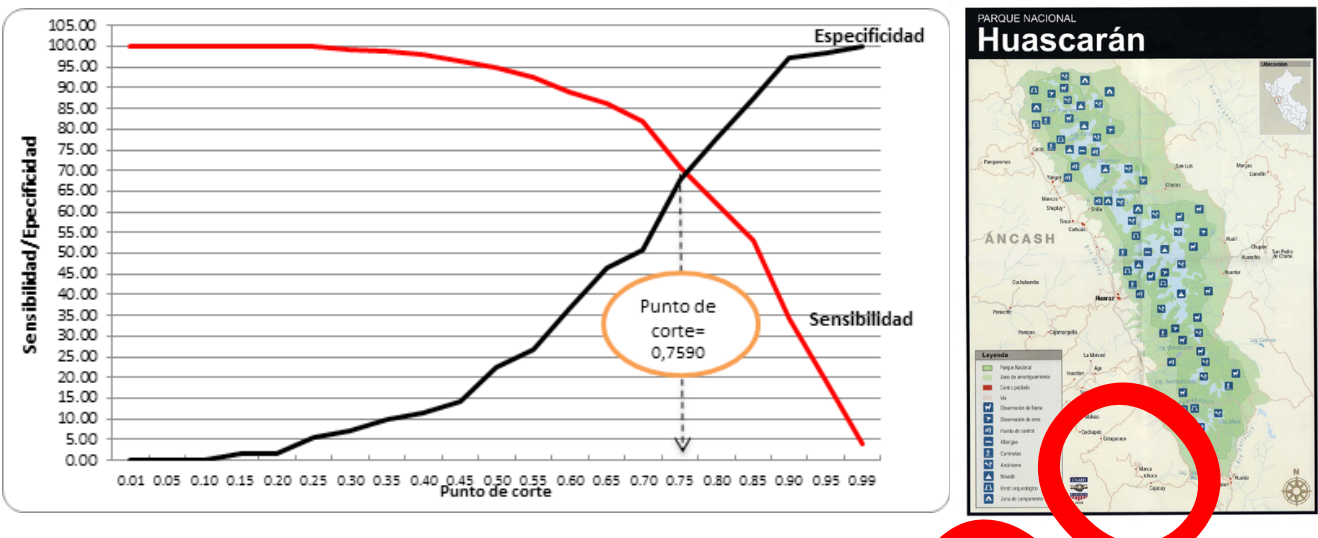

Figura 9. Sensibilidad y especificidad en el punto de c te, y en rapa del PNH

También se constató que los parámetros del indicad ny de mstrucción del jefe de hogar de la ZAPNH son negativos $\left(O R \widehat{\left(\beta_{6}\right)}<1\right)$, lo al significa que, conforme aumentan los niveles de instrucción, los nive es du legrad ción de los recursos naturales focales del Parque, disminuye. Particy nente, s ys jefes de hogar tienen instrucción primaria, el riesgo de degrada ón de las recursos naturales focales disminuye en $11,5 \%$ respecto a la presión ejer ya por s analfabetos. El modelo ajustado fue sometido a la prueba de bon de ajum ue Hosmer y Lemeshow, cuyo estadístico es 4,827 ( $\mathrm{P}=0,7760,05)$ y perm e entror que el modelo de regresión logística es adecuado para relacionar $\mathrm{s}$ 1h licad res de las variables analizadas. En cuanto a la evaluación del riesgo pror stice 1 walde corte es 0,5 y significa que el modelo identifica correctamente 22,5 , te los hogares que no degradan los recursos naturales focales del Parque y $7 \%$ de $>$ hogares que sí lo degradan. En general, la tasa global de calificació $A$ correcta stimada es $78,5 \%$, con $22,5 \%$ para la especificidad y $94,7 \%$ para la sen oflida Para obtener el punto de corte óptimo, con propósitos de clasificación, se selec có el corte maximizador de la sensibilidad y especificidad. El punto ópmo s de 7,9 , intercepto de las curvas de sensibilidad y especificidad, tal como se mu la figura 9. El modelo identifica correctamente al 69\% de hogares que no u. 'izan ningún recurso del Parque y 70,2\% de los hogares que sí utilizan algún recurso.

\section{DISCUSIÓN}

La presente investigación pone de manifiesto que los hogares campesinos de la Zona de Amortiguamiento del Parque Nacional Huascarán son pobres y bajo tal condición generan riesgos de degradación de los recursos naturales focales del Parque Nacional Huascarán, entre los cuales destacan las maderas y matorrales de los bosques y bosquetes demandados como leña para combustible; las hierbas aromáticas y medicinales altoandinas, y las truchas de los ríos y lagunas para el consumo humano; los pastos 
naturales y bofedales por sobrepastoreo del ganado, de modo que coinciden con los hallazgos de Martínez (1991), quien encontró que hay mucha pobreza en el medio rural andino que repercute en la degradación de la frágil foresta por la extracción de la leña y de los pastos naturales en tanto sobrepastoreo; asimismo, con los de (Short et al., 2002) y Méndez (2003), quienes hallaron que la pobreza de las unidades campesinas es un factor esencial en la degradación de los suelos.

Se ha encontrado que hay importantes sectores pobres de la población campesina que padecen de necesidades básicas insatisfechas (servicios de desagüe, alcantarillado, recojo de la basura doméstica, servicios de salud y otras) en la Zona de Amortiguaminnto del Parque. Las personas en edad de trabajar están subocupadas y desocup das en s mayoría y tienen niveles de ingreso económico entre bajos y muy bajos; la actividas económica principal del jefe de hogar es la agricultura; en la cédula la papa, el trigo y el maíz como productos dominantes para el con mo inte o; el nivel educativo prevalente entre los jefes de hogar es la primaria. El min ndis lo sobresale en toda la zona y está asociado con la escasez de tierras ag po arias, a crianza de animales domésticos es mixta y el hato ganadero peque 3 . Toc sto permite afirmar que la economía campesina de dicha zona es de aut co sistenci y se halla poco integrada al mercado. En tal sentido, este trabajo parece confin ar las previsiones teóricas de Chayanov (1974), quien sostiene que la ra onalidad del ampesino pobre apunta a la sobrevivencia y no a la lógica ganancial d mercad, es decir, hay una substancial diferencia de objetivos entre la producción co renc onal y la economía campesina cuyas lógicas productivas son diferente En economía convencional el objetivo del productor es maximizar las utilid y y consumidor, el bienestar; en cambio, en las economías campesinas pol es co no las que existen en la Zona de Amortiguamiento del Parque Nacional H aso ró, er onetivo del cálculo económico es asegurar la subsistencia del grupo viliar.

\section{CONCLUSIC NES}

Es altame ro rob a la pobreza extrema y no extrema prevaleciente entre los hogare camr sinos de la Zona de Amortiguamiento, aproximados por los ingresos económi (y/o necesidades básicas insatisfechas), esté ocasionando riesgos de degradación do os recursos naturales focales del Parque Nacional Huascarán, esto es, de las maderas y matorrales de los bosques demandados como leña, la extracción de hierbas aromáticas y medicinales, así como las truchas de los ríos y lagunas para el consumo del hogar y los pastos naturales y bofedales por sobrepastoreo del ganado.

La relativa escasez de alimentos que sufren los integrantes de los hogares pobres de la Zona de Amortiguamiento del Parque empuja a éstos a practicar la pesca furtiva de truchas en los ríos y las lagunas del Parque poniendo en riesgo su degradación. La escasez de tierras forestales disponibles y los bajos ingresos económicos de los hogares para acceder al uso de energías convencionales como el gas, electricidad o kerosene en 
la ZAPNH conlleva a incursiones clandestinas para la extracción de leña para uso doméstico degradando los bosques, bosquetes y matorrales del Parque. Los hogares con mayores integrantes desocupados aumentan el riesgo de degradación de los recursos naturales focales, respecto de hogares con miembros ocupados.

La educación de los jefes de hogar, aproximada por los años de instrucción, es un factor clave que aminora los riesgos de degradación de recursos naturales focales, pues a mayor nivel de instrucción del jefe de hogar, menor es el riesgo, al punto que un jefe de hogar con instrucción primaria aminora en nueve veces el riesgo de degradación ocasionado por un analfabeto.

\section{AGRADECIMIENTOS}

Nuestra gratitud a la UNASAM, su cuerpo directivo y Iministr ivo por el apoyo financiero; a la administración del PNH y los estudiantes de Far itad de Economía y Contabilidad por viabilizar la aplicación de la Enc esta sum rrse al procesamiento de los datos. Sin el concurso de todos ellos no abría 10 posible concluir este trabajo que está hecho para servir al pueblo peruar or del mur lo entero, empezando por los más pobres.

\section{REFERENCIAS BIBLIOGRAFIO 'S}

Altieri, Miguel y Másera, Omar 19\% Denvolvimento rural sustentanble en América Latina: construindo de baiy pon cim Brasil: Editora da Universidade/UFRGS.

Bartra, Roger. 2010. ntro Chayanov. Brasil: UFPEL. <http://wp.ufpel.edu.br/ consagrg ales/2 0/08/BARTRA-Estudo-sobre-CHAYANOV.pdf $>$ [Consulta: 25 - $3-2$ ( 4$]$.

Chayan , Alexander.1 974. La organización de la unidad económica campesina. Buenos Ais: Yava Visión.

e 1 Paz ot al. 2005. Pobreza Rural y Medio Ambiente. Experiencias en cuatro comu- Iades de la Selva seca de Oaxaca, México. Bogotá: Cuadernos de Desarrollo Rural $\mathrm{N}^{\circ}$ 055. Pontificia Universidad Javeriana. <http://redalyc.uaemex.mx/redalyc/ pdf/117/11705505.pdf> [Consulta: 17-02-2014].

Hernández, Roberto.1994. Teoría sobre campesinado en América Latina: una evaluación crítica. Chile: Revista Chilena de Antropología, N¹2 Santiago de Chile 19931994. 179-300.

INEI. 2009. Mapa de Pobreza Provincial y Distrital 2007: El enfoque de la pobreza monetaria. Lima: Dirección Técnica de Demografía e Indicadores Sociales.

Kustudich, Dan. 2002. Linking poverty reduction and environmental management: policy challenges and opportunities. Washington D.C.: Working Paper, vol. 1, Banco Mundial. 
Martínez Joan. 1991. La pobreza como causa de la degradación ambiental. Barcelona: Universidad Autónoma de Barcelona. Document d'análisi geográfica 18. <http:// www.raco.cat/index.php/DocumentsAnalisi/article/download/41522/52349> [Consulta: 12-01-2014].

Méndez, Juan 2003. Pobreza y biodiversidad. Guatemala. Serie de documentos técnicos No10. Universidad Rafael Landivar, Facultad de Ciencias Ambientales y Agrícolas. <http://www.infoiarna.org.gt/media/File/publicaciones/propias/doc_tecnicos/10-Biodiversidad-Pobreza.pdf $>$ [Consulta: 14-01-2014].

Morales, César y Parada, Soledad. 2005. Pobreza, desertificación y degradación de turales. Capítulo I. Pobreza, desertificación y degradación de tierras. Santiag de Chile Libro 87 CEPAL. Naciones Unidas y GTZ. <http:// repositorio cepal. rg/bits tream/handle/11362/2448/1/S0500967_es.pdf> [Consulta: s-01-2 14].

Short, Clare. et. al. 2002. La vinculación entre la reducción de la pobreza y a vestión mbiental. Retos y opciones de politica estratégica. Reino Unido. Ageno desan o (DFID), Dirección General de Desarrollo de la Comisió Eur pr, Prud y Banco Mundial. <http://www.unpei.depiweb.org/PD Linking overty-red-env-Esp. pdf $>$ [Consulta: 14-03-2014].

Torres, Miguel y Vergara, Sebastián. (s/f). Pobr za, desertificacióny degradación de los recursos naturales. Capítulo III. Proyecto CEPAL GTZ. < ttp://www.eclac.org/publicaciones/xml/8/24268/lcg2277e_fo III.__e Lonsulta: 10-03-2014].

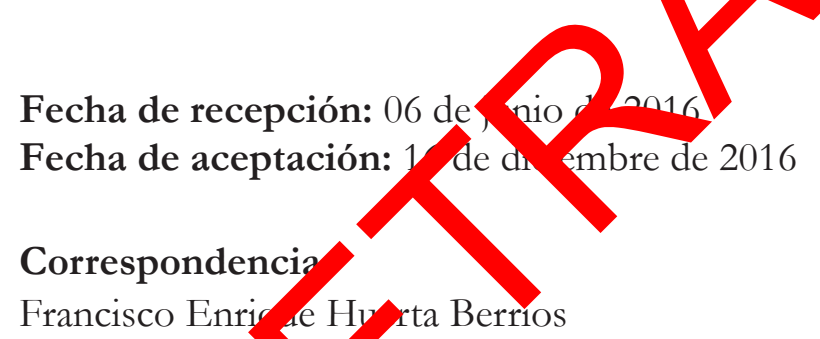

E-mail: ehuertab otmain com 